\title{
Genetic platelet depletion is superior in platelet transfusion compared to current models
}

Haematologica 2020

Volume 105(6):1738-1749

\section{Correspondence:}

ALICE ASSINGER

alice.assinger@meduniwien.ac.at

Received: March 19, 2019.

Accepted: September 19, 2019.

Pre-published: September 19, 2019.

doi:10.3324/haematol.2019.222448

Check the online version for the most updated information on this article, online supplements, and information on authorship \& disclosures: www. haematologica.org/content/105/6/1738

(C)2020 Ferrata Storti Foundation

Material published in Haematologica is covered by copyright. All rights are reserved to the Ferrata Storti Foundation. Use of published material is allowed under the following terms and conditions:

https://creativecommons.org/licenses/by-nc/4.0/legalcode. Copies of published material are allowed for personal or internal use. Sharing published material for non-commercial purposes is subject to the following conditions:

https://creativecommons.org/licenses/by-nc/4.0/legalcode, sect. 3. Reproducing and sharing published material for commercial purposes is not allowed without permission in writing from the publisher.

\author{
Manuel Salzmann, ${ }^{1}$ Waltraud C. Schrottmaier, ${ }^{1}$ Julia B. Kral-Pointner, ${ }^{1}$ \\ Marion Mussbacher, ${ }^{1}$ Julia Volz, ${ }^{3}$ Bastian Hoesel, ${ }^{1}$ Bernhard Moser, ${ }^{1}$ \\ Sonja Bleichert, ${ }^{1,2}$ Susanne Morava, ${ }^{1}$ Bernhard Nieswandt, ${ }^{3}$ \\ Johannes A. Schmid ${ }^{1}$ and Alice Assinger ${ }^{1}$
}

${ }^{1}$ Institute of Vascular Biology and Thrombosis Research, Medical University of Vienna, Vienna, Austria, ${ }^{2}$ Department of Surgery, General Hospital, Medical University Vienna, Vienna, Austria and ${ }^{3}$ Institute of Experimental Biomedicine, University Hospital and Rudolf Virchow Center, University of Würzburg, Würzburg, Germany

\section{ABSTRACT}

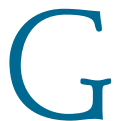
enetically modified mice have advanced our knowledge on platelets in hemostasis and beyond tremendously. However, mouse models harbor certain limitations, including availability of platelet specific transgenic strains, and off-target effects on other cell types. Transfusion of genetically modified platelets into thrombocytopenic mice circumvents these problems. Additionally, ex vivo treatment of platelets prior to transfusion eliminates putative side effects on other cell types. Thrombocytopenia is commonly induced by administration of anti-platelet antibodies, which opsonize platelets to cause rapid clearance. However, antibodies do not differentiate between endogenous or exogenous platelets, impeding transfusion efficacy. In contrast, genetic depletion with the inducible diphtheria toxin receptor (iDTR) system induces thrombocytopenia via megakaryocyte ablation without direct effects on circulating platelets. We compared the iDTR system with antibody-based depletion methods regarding their utility in platelet transfusion experiments, outlining advantages and disadvantages of both approaches. Antibodies led to thrombocytopenia within two hours and allowed the dose-dependent adjustment of the platelet count. The iDTR model caused complete thrombocytopenia within four days, which could be sustained for up to 11 days. Neither platelet depletion approach caused platelet activation. Only the iDTR model allowed efficient platelet transfusion by keeping endogenous platelet levels low and maintaining exogenous platelet levels over longer time periods, thus providing clear advantages over antibody-based methods. Transfused platelets were fully functional in vivo, and our model allowed examination of transgenic platelets. Using donor platelets from already available genetically modified mice or ex vivo treated platelets, may decrease the necessity of platelet-specific mouse strains, diminishing off-target effects and thereby reducing animal numbers.

\section{Introduction}

Platelets are anucleate cells, which derive from bone marrow megakaryocytes. Beyond their central role in hemostasis, platelets fulfill important functions in inflammation and infection, ${ }^{1}$ atherogenesis ${ }^{2}$ and tissue regeneration. ${ }^{3}$ Genetically modified mice are valuable tools to investigate the role of platelet function in hemostasis and beyond in complex in vivo systems. However, ascertaining the specific contribution of platelets in global knockout mice can be challenging. Usage of the tissue-specific Cre/loxP system is one possibility to circumvent this problem and enables the investigation of a genetic modification in selected cell types. Mouse lines expressing Cre recombinase via the platelet- and megakaryocyte-specific platelet factor $4(\mathrm{PF} 4)^{4}$ or the glycoprotein (GP) $1 \mathrm{~b} \alpha$ promoters $^{5}$ are essential to delineate distinct platelet mediated effects in complex physiological and pathophysiological settings. However, crossing of mice is expensive and time-consuming 
and loxP flanked genes of interest are not available in all cases.

An alternative to overcome these limitations is transfusion of genetically or pharmacologically modified platelets into platelet depleted mice. This allows research of altered platelets in a genetically unchanged environment. Additionally, platelets can be treated ex vivo before transfusion, eliminating putative side effects of the treatment on other cell types. Platelet depletion prior to platelet transfusion is frequently performed by administration of antibodies targeting platelet-specific epitopes although this harbors certain inherent limitations: Platelets are opsonized by antibodies and actively scavenged from the circulation. This could lead to immune reactions or activation of opsonized and agglomerated platelets. Most importantly though, antibody binding does not differ between endogenous and transfused platelets, which either negates transfusion or demands repeated cycles of depletion and transfusion, to maintain a stable population of exogenous platelets.

A solution to this limitation is provided by a novel model, in which an inducible diphtheria toxin (DT) receptor (iDTR) is expressed under the control of a platelet-specific PF4 Cre recombinase, rendering megakaryocytes susceptible to DT-induced termination of protein synthesis. ${ }^{6,7}$ A single DT molecule in the cytoplasm is sufficient to kill an iDTR-expressing cell, ${ }^{8}$ whereas wild-type (WT) murine cells are highly insensitive towards DT, making megakaryocyte ablation exceptionally specific and efficient.

Here, we aimed to validate the platelet iDTR model and compared it with antibody-mediated platelet depletion methods regarding its use in platelet transfusion experiments. We show for the first time that the iDTR model can be successfully used in platelet transfusion and that it provides several advantages over antibody-mediated depletion by simplifying the experimental setup and refining platelet transfusion. Additionally, the iDTR model may help to avoid time consuming generation of tissuespecific mice and allows analysis of platelet-specific functions, in cases where only a full knockout of the gene of interest is available.

\section{Methods}

Detailed methods regarding genotyping, platelet function assays, and in vivo experiments can be found in the Online Supplementary Materials and Methods.

\section{Mice}

All experiments and animal studies were conducted according to institutional guidelines and were approved by the Animal Care and Use Committee of the Medical University of Vienna (BMWFM-66.009/0246-WF/V/3b/2016).

All experimental procedures were conducted according to the SYRCLE's risk of bias tool for animal studies; e.g. groups were age matched littermates, and after initial scouting experiments, only female mice were used in all studies, to minimize a risk of bias; animals within the same cage were preferably taken into the same experiment; cages of $\mathrm{iDTR}^{\mathrm{Plt}}$ mice were randomly selected for experiments; blinding was not applicable.

\section{Platelet depletion}

Megakaryocyte and consequently platelet depletion were induced in iDTRPlt mice by subcutaneous injections of $100 \mathrm{ng}$ DT on days 0,2 and 4, followed by 250 ng on days 7, 9 and 11 . To prevent hematoma and scarring, mice were anesthetized with isoflurane (Abbot Laboratories) for subcutaneous injections after day 7. WT mice were platelet depleted by intravenous administration of $4 \mu \mathrm{g} / \mathrm{g}$ anti-mouse GPIb $\alpha$ (R300, Emfret Analytics), $0.2 \mu \mathrm{g} / \mathrm{g}$ antimouse platelet monoclonal antibody 6A6-IgG2A (originating from Dr R. Good, University of South Florida College of Medicine, Tampa, FL, USA) or intraperitoneal injection of $15 \mu \mathrm{L}$ rabbit anti-mouse thrombocyte serum (AIA31440, Accurate Chemical \& Scientific Corporation).

\section{Platelet transfusion}

DT treatment was started at day 7 and R300 treatment 12 hours prior to transfusion. Recipients were injected with $4.3 \times 108$ washed platelets from WT or naive male iDTRPlt mice, labeled with anti GPIb $\beta$ DyLight649 (X649, Emfret Analytics) and blood samples were collected after 2, 14, 48 and 72 hours. Blood was labeled with anti-CD41-BV421 and analyzed using a CytoflexS flow cytometer with Cytexpert 2.2 software. Exogenous platelets were defined as $\mathrm{CD}_{4} 1^{+} \mathrm{X} 649^{+}$events, whereas endogenous platelets were $\mathrm{CD}_{4} 1^{+} \mathrm{X} 649^{-}$.

\section{Statistics}

Calculations were performed using GraphPad Prism 8.02 software. Statistical significances are depicted as: ${ }^{*} P \leq 0.05,{ }^{* *} P \leq 0.01$, ${ }^{* * *} P \leq 0.001,{ }^{* * * *} P \leq 0.0001$.

Comparison of two groups was done by unpaired t-test or Mann-Whitney test if data was not distributed normally. Two or more groups were compared to the respective control group using One-Way ANOVA with Dunnett correction. If all groups were compared with each other, Tukey correction was applied. Two groups with more than one condition were compared by TwoWay ANOVA and Sidak correction. For Figure 1D-E, H-I and Figure 4B-E individual area under the curves (AUC) were calculated and groups compared to the respective control using One-Way ANOVA with Dunnett correction.

\section{Results}

\section{Selective killing of megakaryocytes leads to rapid platelet depletion}

To selectively ablate megakaryocytes and induce thrombocytopenia, we crossed mice expressing iDTR downstream of a loxP-flanked stop cassette with a PF4 iCre strain to generate mice with megakaryocytes expressing iDTR (iDTR ${ }^{\text {Plt }}$ ) (Figure 1A). Naïve iDTR ${ }^{\text {Plt }}$ mice did not show any obvious alterations in bone marrow or megakaryocyte morphology (Online Supplementary Figure $S 1 A$ ), nor was the megakaryocyte count affected (Online Supplementary Figure S1B). For platelet depletion, we administered DT in a weekly routine with $100 \mathrm{ng}$ being injected subcutaneously on days 0,2 and 4 . The dosage was increased at day 7 to 250 ng to avoid rebound effects and maintain efficient thrombocytopenia (Figure 1B), as megakaryocyte count return to basal levels within two days after last $100 \mathrm{ng}$ injection (Online Supplementary Figure S1C). As controls we injected PBS into iDTR ${ }^{\mathrm{Plt}}$ mice and DT into WT mice. Megakaryocytes were already remarkably depleted on day 2 (Online Supplementary Figure S1D) and undetectable from day 4 till day 7 (Figure 1C). Platelet count decreased over time in DT-treated $i_{D T R}{ }^{\text {Plt }}$ mice, reaching a nadir with day 4 , and remained low until day 12 (Figure 1D). Platelet counts of control groups remained unaffected. In parallel, mean platelet size increased in the 

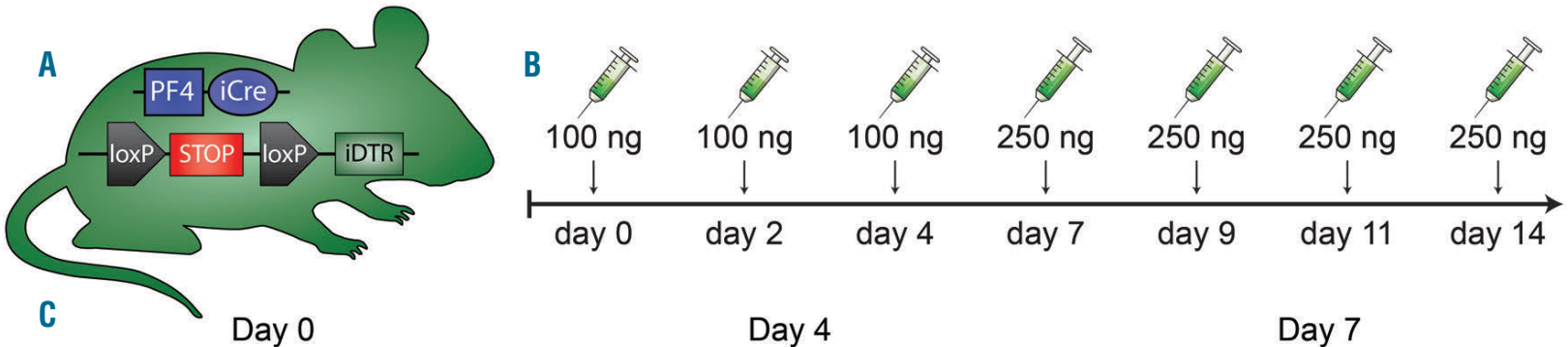

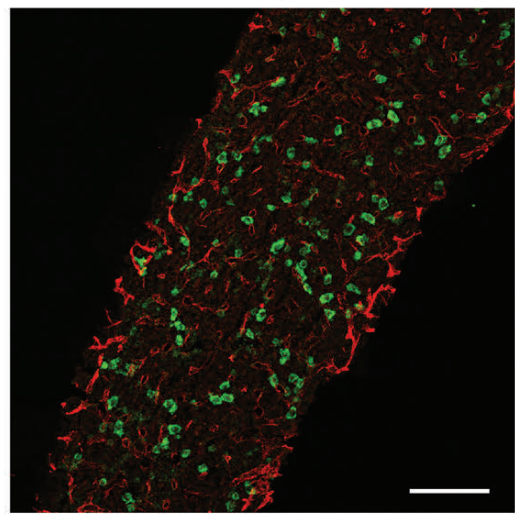

Day 4

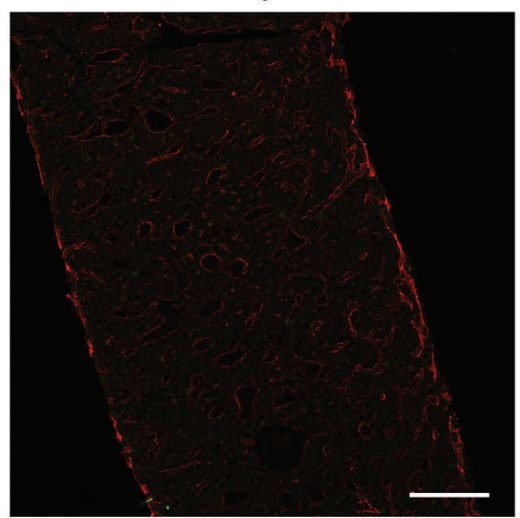

CD41 endomucin

D Platelet count

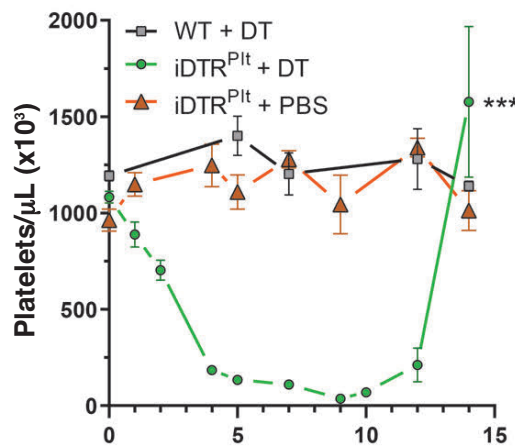

Days after initial injection

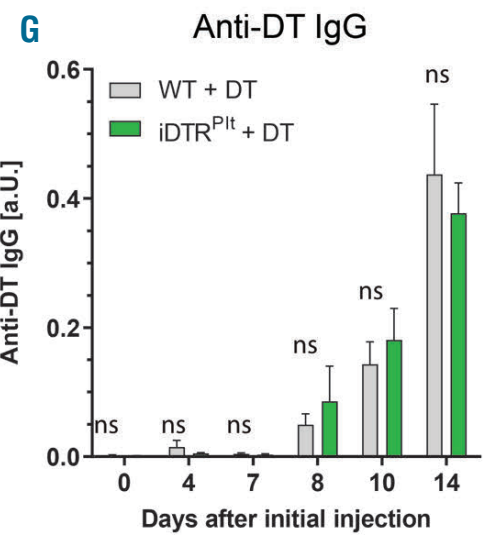

E

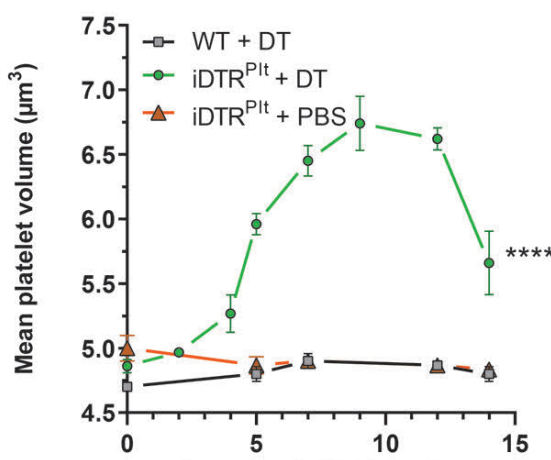

Days after initial injection

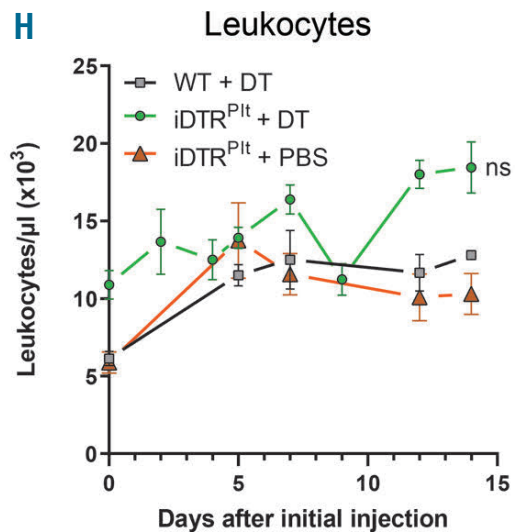

Day 7
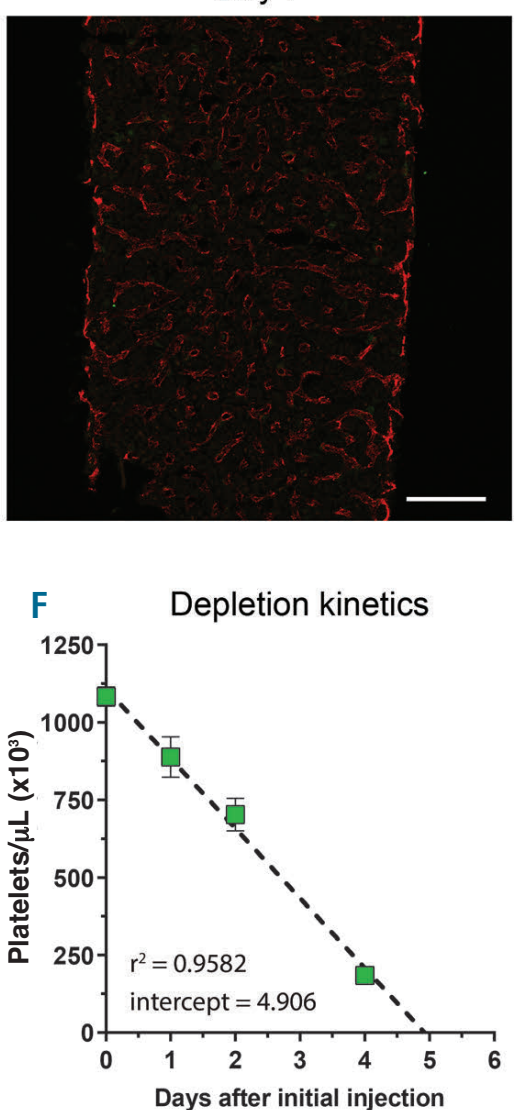

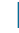

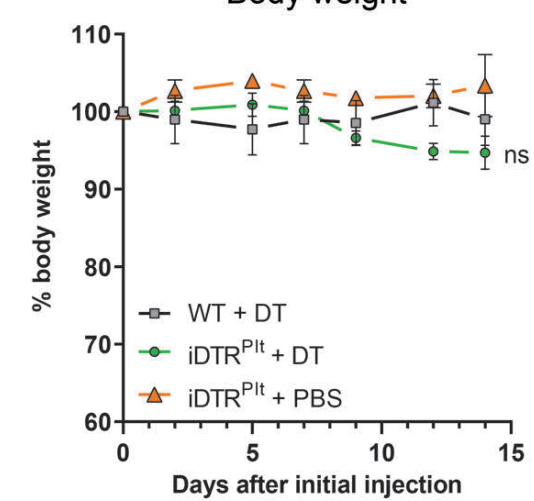

Figure 1. Depletion of megakaryocytes and platelets with diphtheria toxin. (A) Genetic scheme of PF4-iCre iDTR mice, termed iDTR ${ }^{\text {plt }}$ mice. (B) Graphical overview of diphtheria toxin (DT) administration scheme. (C) Femora of untreated and mice treated for four and seven days with DT. Bone marrow was stained for endothelial cells (endomucin, red) and megakaryocytes (CD41, green). Scale bar: 200 um. (D) Platelet counts and (E) mean platelet volume after indicated days of DT treatment. (F) Linear regression of decrease in platelet counts of iDTR ${ }^{\text {Plt }}$ mice within first four days of DT treatment. 95\% confidence interval of X-intercept: 4.563-5.318. (D-F) Curves of groups were compared to that of iDTR ${ }^{\mathrm{ptt}}+\mathrm{DT}(\mathrm{n}=3-8)$. $(\mathrm{G})$ Titer of anti-DT IgG after indicated times of treatment, normalized to a DT-boosted mouse ( $\mathrm{n}=4$ 17). (H) Leukocyte counts and (I) body weight after indicated days of DT treatment. (H-I) Curves of groups were compared to that of iDTR ${ }^{\text {Plt }}+$ DT ( $\left.n=3-8\right)$ 
remaining platelet population during DT treatment (Figure $1 E)$. Notably, linear regression of platelet counts until day 4 revealed that their decrease corresponded to their reported lifespan (Figure 1F) of 4.8 days. ${ }^{10}$ Platelets were therefore not directly affected by DT treatment and thrombocytopenia was induced by megakaryocyte depletion rather than platelet ablation. Examining a putative immune response against DT, we quantified DT-specific immunoglobulin G (IgG) generated during treatment. Antibodies were detectable from day 8 onwards, with no significant difference between $\mathrm{iDTR}^{\mathrm{Plt}}$ and WT mice, indicating that presence of iDTR on platelets did not alter the adaptive immune response (Figure 1G). Furthermore, leukocyte count remained unaffected in thrombocytopenic iDTR ${ }^{\mathrm{Plt}}$ mice (Figure $1 \mathrm{H}$ ). Upon prolonged platelet depletion mice displayed an increased burden of hematomata and impaired wound healing, with body weight being slightly, but not significantly decreased (Figure 1I). We also observed that male mice fared worse than females (data not shown). Therefore, only female mice were used in further experiments.

\section{Presence of IDTR does not affect platelet function}

To monitor potential off-target effects of iDTR expression on platelet physiology, we performed blood counts as well as platelet function assays. Untreated iDTR $^{\mathrm{Plt}}$ mice displayed normal blood cell counts with no significant differences in platelet, leukocyte and erythrocyte numbers compared to WT mice (Figure 2A-C) although mean platelet volume (MPV) was slightly increased (Figure 2D). However, this did neither affect the hemostatic platelet function, nor the interaction with leukocytes. Light transmission aggregometry revealed no alterations in aggregation upon stimulation with thrombin or collagen I (Figure $2 \mathrm{E}-\mathrm{F}$ and Figure 3D). Further, platelet activation was evaluated by stimulating the major platelet activation receptors PAR4 with PAR4-activating peptide (PAR4-AP), GPVI with convulxin (CVX), and $\mathrm{P}_{2} \mathrm{Y}_{1 / 12}$ with ADP (Figure $3 \mathrm{~A}$ ). Expression of iDTR on platelets did not affect surface expression of the degranulation marker P-selectin (CD62P) when compared to WT littermate controls (Figures 3B), nor did it influence activation of GPIIb/IIIa (Figure 3C). As an example for non-classical functions of platelets, we evaluated their ability to form aggregates with monocytes and neutrophils which was also unaltered by iDTR expression (Figure 3E-H). These data indicate that physiological platelet function is preserved in naive $\mathrm{iDTR}^{\mathrm{Plt}}$ mice.

\section{Comparison of platelet depletion strategies}

Next, we compared the iDTR model with antibodybased depletion methods in terms of platelet activation. Blood samples were taken prior to platelet depletion

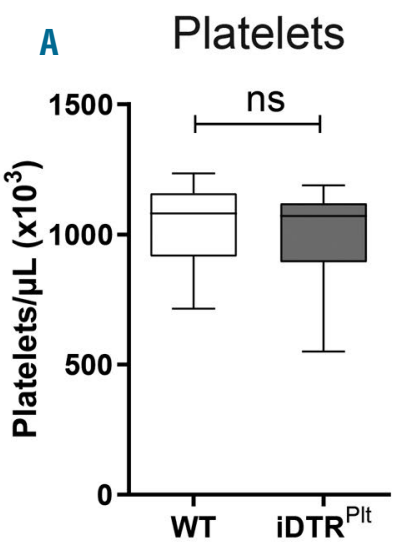

D Platelet size

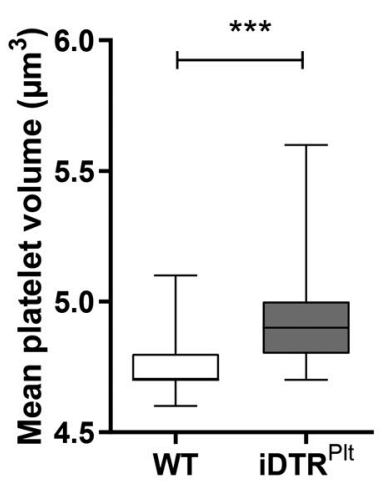

B Leukocytes

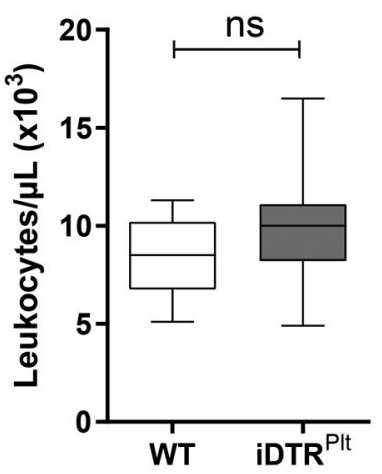

E Thrombin

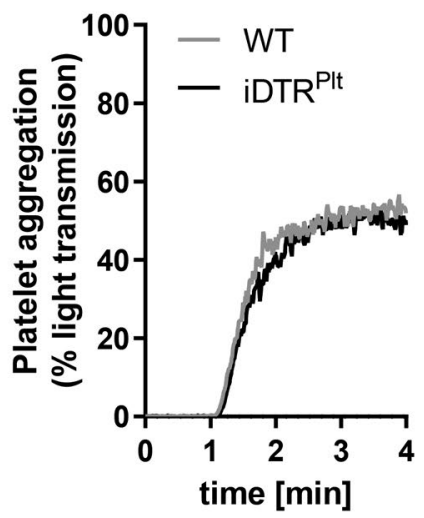

c Erythrocytes

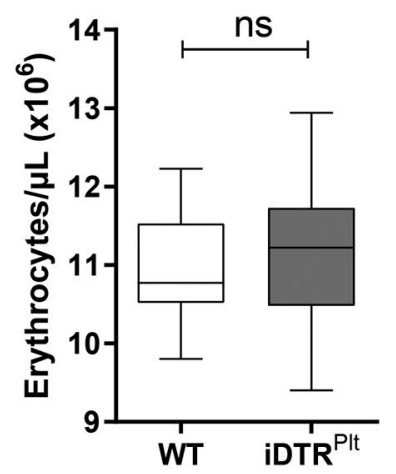

F Collagen

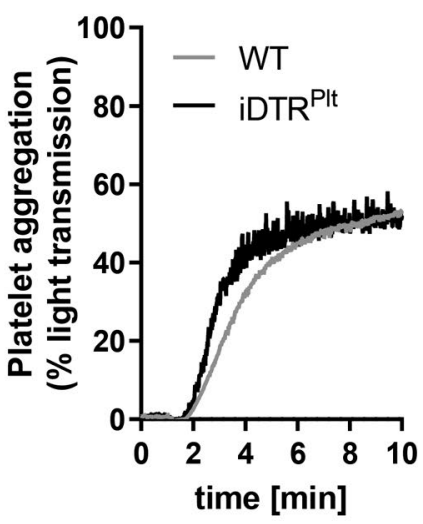

Figure 2. iDTR expression does not alter blood counts or platelet aggregation. Comparison of blood count: (A) platelets, (B) leukocytes, (C) erythrocytes and (D) mean platelet volume $(n=13-14)$. Representative aggregation curves of washed platelets stimulated with thrombin $(E)$ or $(F)$ collagen I. 
A
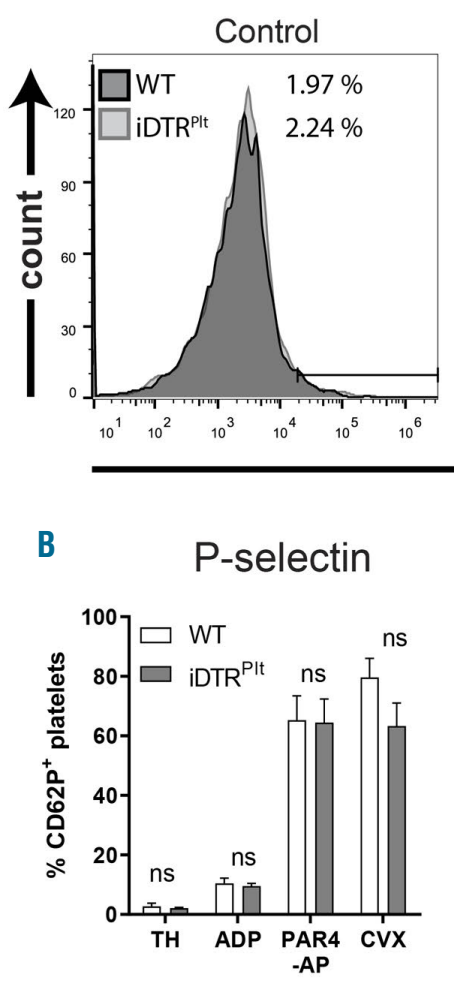

Platelet activation

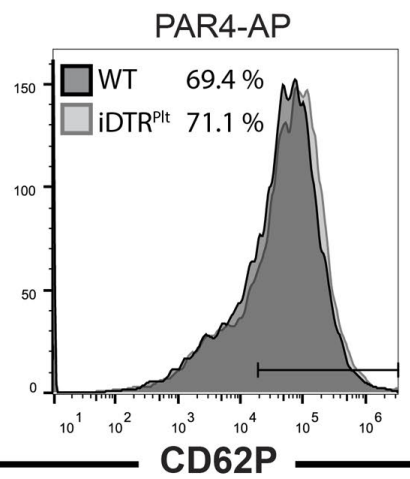

C

act. GPIIb/IIla

D

Aggregation
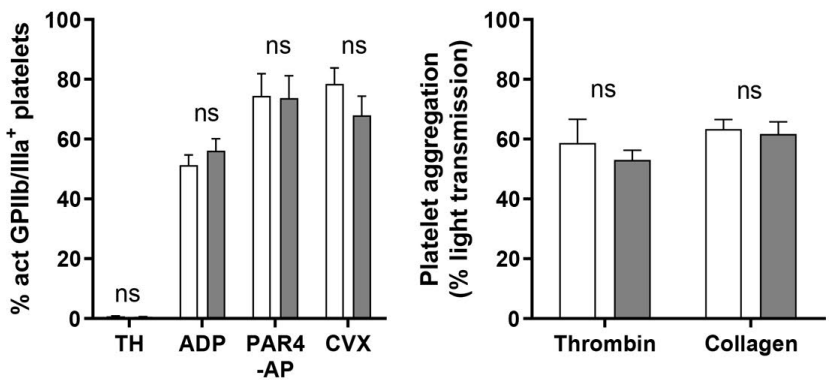

E

Platelet-leukocyte aggregates
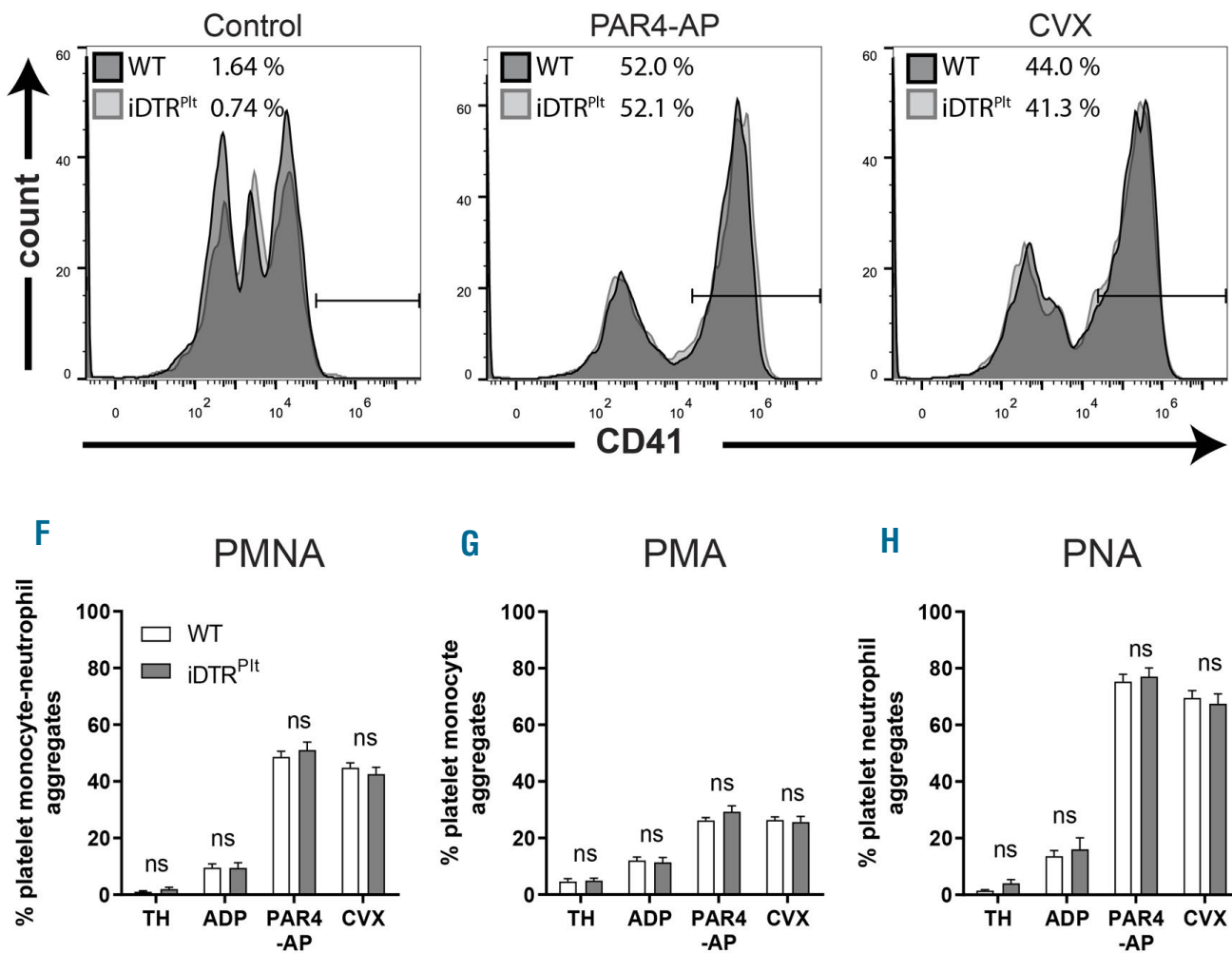

G

PMA

H

PNA
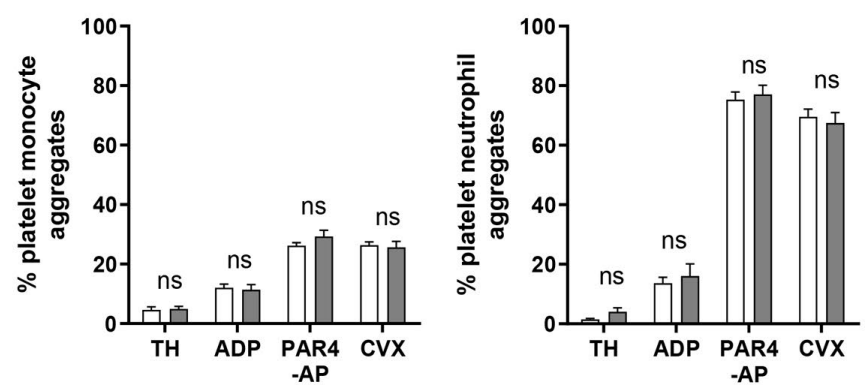

Figure 3. Expression of iDTR does not change platelet function. (A) Representative histograms showing CD62P expression of CD41 ${ }^{+}$cells in diluted whole blood, stimulated with PAR4 agonist peptide (PAR4-AP), or convulxin (CVX), or treated with Tyrode's HEPES buffer (TH). (B) Percentage CD62 ${ }^{+}$and $(\mathrm{C})$ activated GPIIb/IIla ${ }^{+}$ of $C D 41^{+}$events in diluted whole blood, stimulated with ADP, PAR4-AP or CVX ( $\left.n=7-8\right)$. (D) Light transmission aggregometry of washed platelets stimulated with thrombin or collagen I $(n=3-5)$. (E) Representative histograms of CD41 expression of CD11 $b^{+}$, CD45 ${ }^{+}$events in blood, stimulated with PAR4-AP or CVX. (F) Percentage CD41 of $\mathrm{CD}_{11} \mathrm{~b}^{+}, \mathrm{CD} 45^{+}$events in whole blood, stimulated with ADP, PAR4-AP or CVX. (G) Sub classification into CD41 side scatter medium monocytes and (H) side scatter high neutrophils $(n=8)$. 
induction and two days or two hours after the first DT or antibody/rabbit anti-mouse thrombocyte serum treatment, respectively. Additionally, platelet activation was assayed at time points of stable platelet depletion, i.e. seven days after DT injection or 12 hours after antibody injection, as well as three days afterwards (Figure 4A). Different anti-platelet antibodies were applied and compared with the iDTR system: while the antibodies anti-GPIbo (R300) and 6A6-IgG2A rapidly induced stable thrombocytopenia of different degree within two hours, anti-mouse thrombocyte serum gradually reduced platelet counts to $10.8 \pm 9.8 \%$ within 12 hours (Figure 4B). Depletion with antibodies or serum did not significantly increase the expression of P-selectin on the remaining platelets, but seven days after first DT administration, the remaining $0.2 \pm 0.2 \%$ platelets of $\mathrm{iDTR}^{\mathrm{Plt}}$ mice showed slightly increased reactivity (Figure 4C). However, in comparison with a positive control of PAR4-AP stimulated blood, platelet activation in iDTRPlt mice was still minor. As expected, thrombocytopenia resulted in decreased plasma CXCL4 concentrations compared to basal levels when using R300, serum or the iDTR model (Figure 4D), underlining the lack of platelet activation upon induced thrombocytopenia. This is also reflected by normal platelet-leukocyte-aggregate (PLA) formation (Figure 4E).

\section{The IDTR model outperforms antibody-based platelet depletion in transfusion studies}

As a next step, we compared the iDTR model with antibody-based platelet depletion as a potential tool for platelet transfusion. DT treatment started seven days and R300 (4 $\mu \mathrm{g} / \mathrm{mL})$ was administered 12 hours prior to trans-
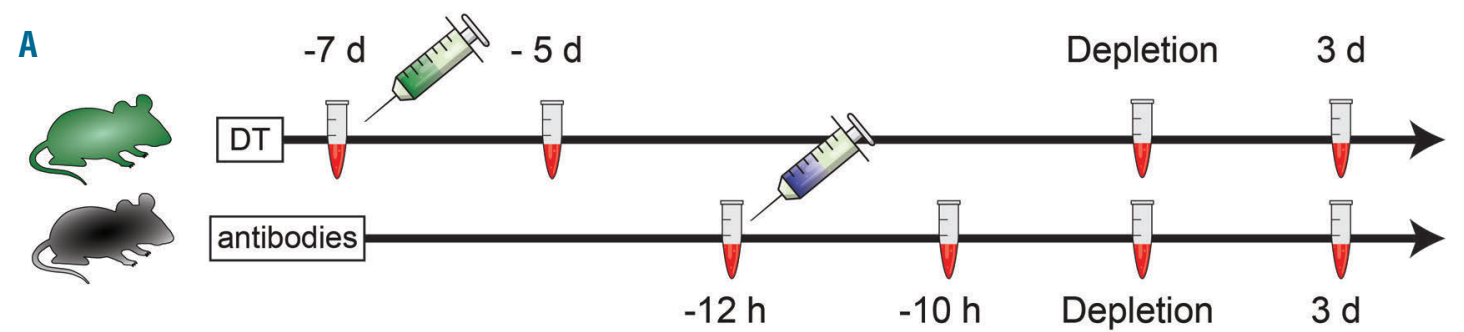

B

Platelet count

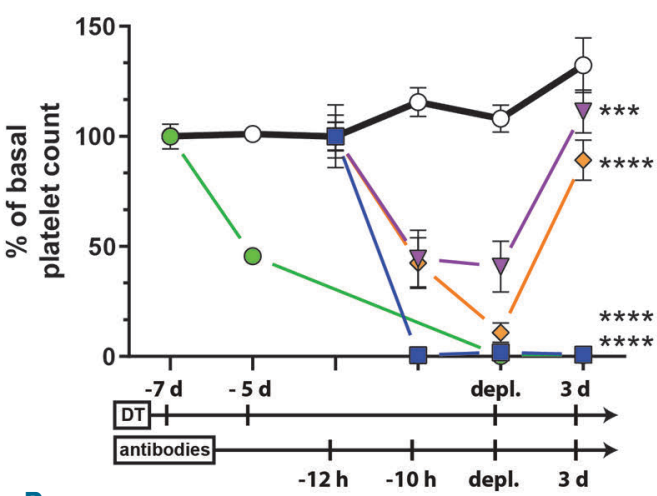

D

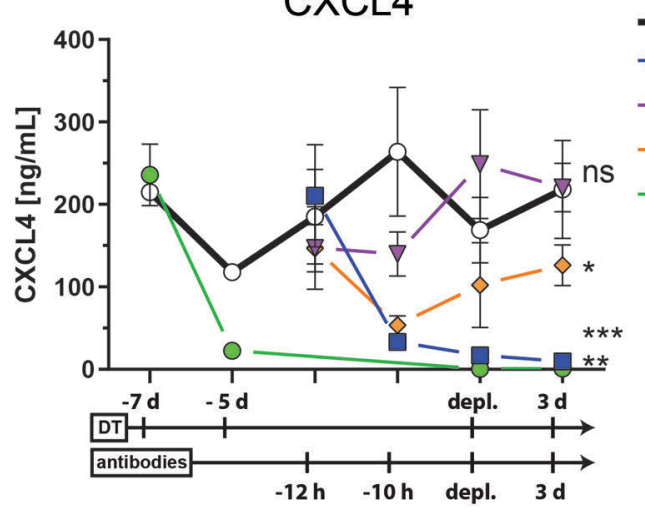

C

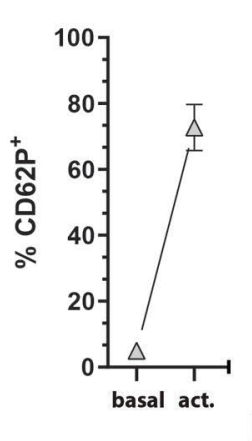

P-selectin

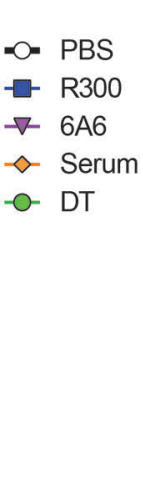

$\mathbf{E}$

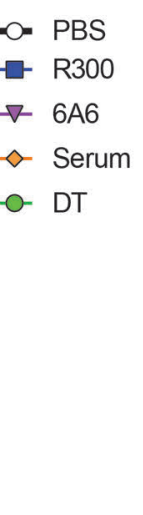

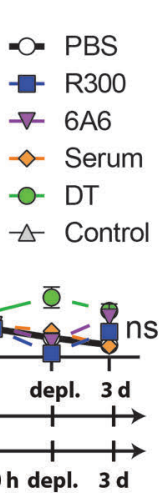

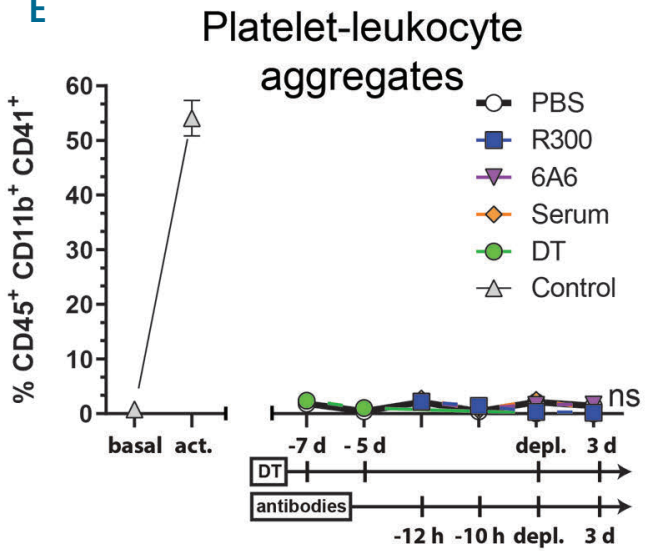

Figure 4. Neither antibody-based nor diphtheria toxin-induced thrombocytopenia cause platelet activation. (A) Graphical overview of depletion comparison. Diphtheria toxin (DT) treatment started seven days (d) prior to stable depletion and R300 treatment 12 hours (h) prior to stable depletion. Blood sampling time points are indicated as reaction tubes. (B) Percentage of platelet counts, relative to initial counts. (C) Percentage CD62P ${ }^{+}$of CD41 ${ }^{+}$events in diluted whole blood, with PAR4AP-activated positive controls. (D) CXCL4 plasma concentrations. (E) Percentage CD41+ of CD11 $\mathrm{b}^{+}$, CD45+ events in whole blood, with PAR4-AP activated positive controls $(n=4-5)$. 
fusion. Efficacy of transfusion was analyzed 2, 24, 48 and 72 hours after platelet injection (Figure 5A). To differentiate between endogenous and transfused platelets, platelets were labeled ex vivo with a fluorescent antibody directed against murine GPIb $\beta$. Platelet counts dropped to $0.2 \pm 0.05 \%$ of baseline in $\mathrm{iDTR}^{\mathrm{Plt}}$ mice and to $0.5 \pm 0.2 \%$ in R300-treated mice. Theoretically, transfusion of all platelets, calculated by estimating a blood volume of 77 $80 \mu \mathrm{L} / \mathrm{g}$ mouse, ${ }^{11,12}$ would have reached $62.5-65 \%$ of basal platelets counts. In $\mathrm{iDTR}^{\mathrm{Pt}}$ mice, platelet transfusion raised platelet counts to $34.8 \pm 19.2 \%$ after two hours, whereas $33.6 \pm 18.7 \%$ of platelets were of exogenous origin. In contrast, platelet counts of R300-treated mice remained at $0.5 \pm 0.2 \%$ after transfusion, with $0.1 \pm 0.1 \%$ of platelets being of exogenous origin. Counts of endogenous platelets remained stable in $\mathrm{DDTR}^{\mathrm{Plt}}$ mice, while R300 treatment was unable to maintain thrombocytopenia at day 3 (Figure 5B). Lower concentrations of R300 mitigated depletion and enhanced transfusion efficacy, indicating that excess antibody removed newly transfused platelets

A

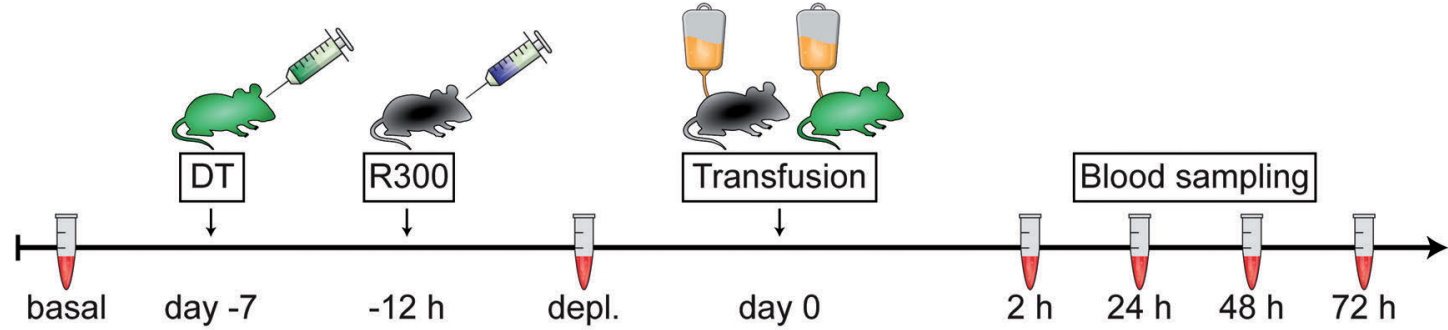

Bi Total platelet count
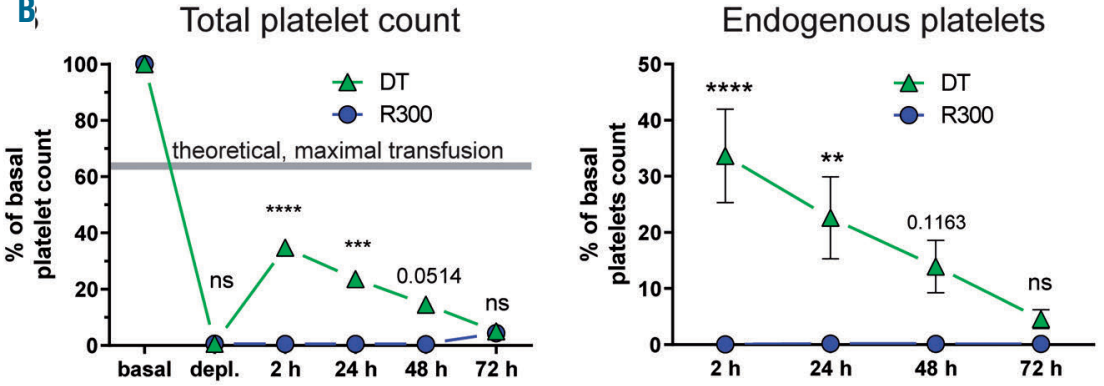

Transfused platelets

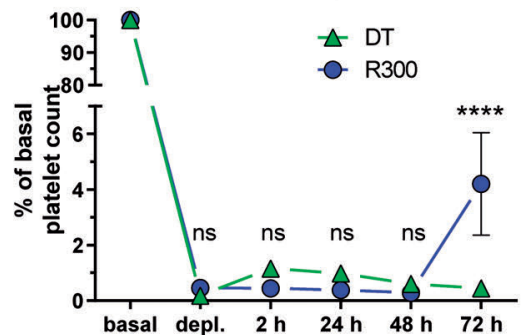

C
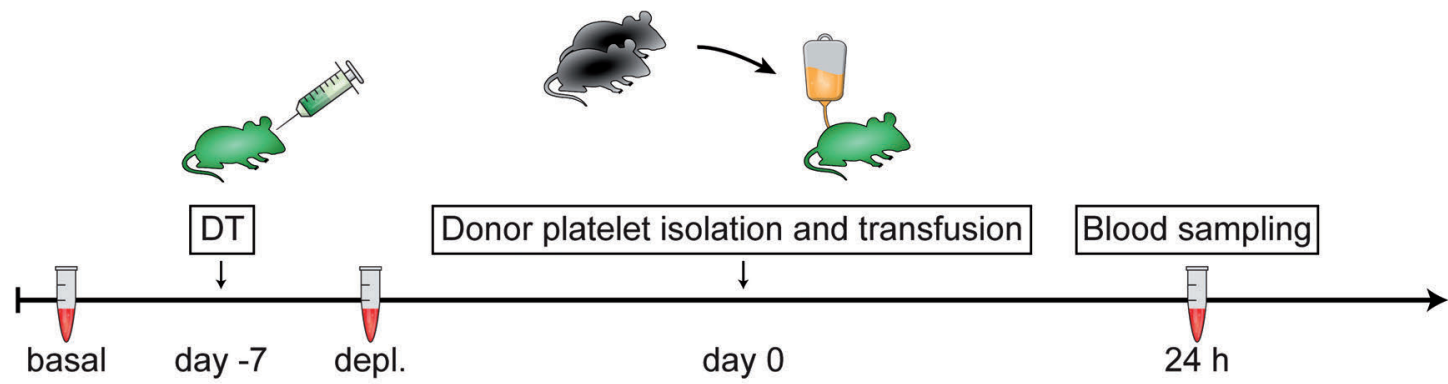

D

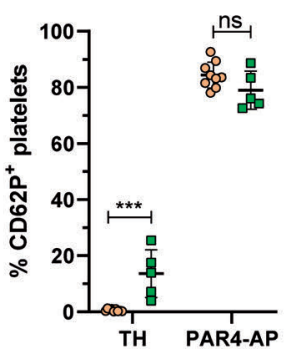

Platelet function

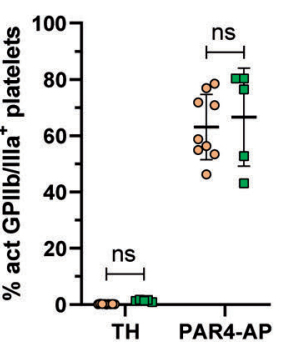

$E$

Level of activation

- Donor - iDTR $^{\mathrm{PIt}} 24 \mathrm{~h}$

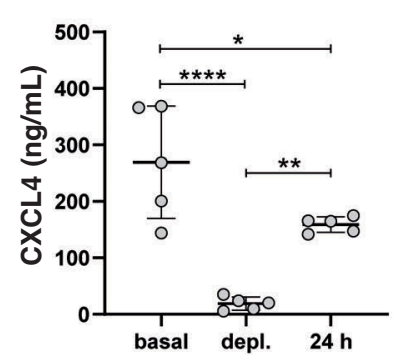

Concentration of

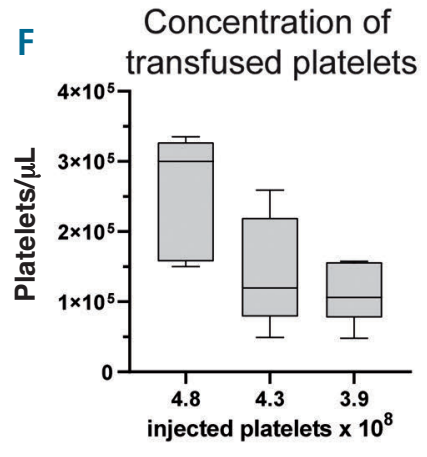

Figure 5. Platelet transfusion efficacy and donor platelet function analysis of iDTR ${ }^{\text {plt }}$ mice. (A) Graphical overview for comparison of platelet transfusion. Diphtheria toxin (DT) treatment started seven days prior to transfusion and R300 treatment 12 hours (h) prior to transfusion. Blood was taken at basal and depleted state, and 2, 24, 48 and 72 hours after transfusion. (B) Percentage of total, endogenous and transfused platelet counts, relative to initial counts. Transfused platelets were labeled with an anti-GPIb $\beta$-Dylight649 antibody. Theoretical, maximal transfusion is depicted as grey area $(n=5)$. (C) Graphical overview of donor platelet function evaluation. DT treatment started seven days prior to transfusion and blood was taken at basal and depleted state, and 24 hours after transfusion. (D) Comparison of percentage of $\mathrm{CD}_{2} \mathrm{P}^{+}$and activated GPIIb/IIla ${ }^{+}$platelets in whole blood, freshly drawn from donors and after circulating for 24 hours in iDTR ${ }^{\text {Ptt }}$ mice $(n=4-9)$. ( $E$ ) Concentration of plasma CXCL4 of iDTR ${ }^{\text {Plt }}$ mice at basal and depleted levels, and 24 hours after platelet transfusion $(n=5)$. (F) Concentration of circulating exogenous platelets after transfusion of indicated numbers of platelets $(n=5-10)$. 
(Online Supplementary Figure S2A-B). Platelet isolation did not lead to increased basal activation but reduces the responsiveness of washed platelets to PAR4 stimulation as compared to whole blood (Online Supplementary Figure S2C). These data indicate that genetic ablation of platelets results in a much higher platelet transfusion efficacy. In antibody-mediated models, even upon adjustment of platelet depletion, transfusion efficacy is lower and purity of circulating exogenous platelets cannot be maintained.

\section{Transfused platelets are fully functional}

Next, we examined the function of transfused platelets. The activation status and platelet reactivity of donor platelets was analyzed immediately after blood drawing and 24 hours after transfusion into platelet-depleted iDTR $^{\text {Plt }}$ mice (Figure 5C). While donor platelets showed low levels of platelet activation marker (surface expression of CD62P and GPIIb/IIIa activation) before transfusion, surface expression of CD62P was slightly - though significantly- increased 24 hours after transfusion. In contrast, GPIIb/IIIa activation remained at the initial level (Figure 5D). Donor platelets were fully responsive to PAR4 stimulation after 24 hours of circulation (Figure 5D). In addition, we evaluated plasma levels of CXCL4 to indirectly determine indications for in vivo platelet activation. As shown in Figure 4D, depletion of platelets led to a profound reduction of CXCL4 in plasma. Transfusion of platelets increased CXCL4 concentration although it did not reach basal levels, which can be explained by the reduced number of platelets. Noteworthy, basal CXCL4 levels with each mouse's endogenous platelets, had a higher variation than those 24 hours later where all iDTR ${ }^{\text {Plt }}$ mice received platelets from the same pool (Figure 5E). These data indicate that transfused platelets did not become activated in vivo. Of note, concentrations of transfused platelets slightly differed between experiments, and higher concentrations of donor platelets were always reflected by higher platelet counts (Figure 5F). Therefore, concentration of transfused platelets can be adjusted in the circulation, dependent on the number of platelets injected.

\section{Transfused platelets are fully functional in vivo}

Finally, we verified that transfused platelets are fully functional in vivo by analyzing their function in mouse models of inflammation and thrombosis. Moreover, we verified the applicability of our model for examination of genetically modified platelets. First, we checked if platelet transfusion can rescue macrophage recruitment in thrombocytopenic iDTR ${ }^{\mathrm{Pt}}$ mice upon sterile inflammation. After seven days of DT treatment iDTR ${ }^{\text {Plt }}$ mice received platelet transfusion, and peritonitis was induced by thioglycollate injection. Three days later, peritoneal macrophages were isolated, and the number of recruited cells was compared between WT, iDTR ${ }^{\text {Plt }}$ and iDTR $^{\text {Plt }}$ mice that received platelet transfusion (Figure 6A). Compared to WT mice, thrombocytopenic $\mathrm{iDTR}^{\mathrm{Plt}}$ mice showed a significant reduction in recruited peritoneal leukocytes; the majority were F4/80 positive macrophages, which was restored by platelet transfusion. In thrombocytopenic mice, leukocyte recruitment was reduced to $30.9 \%$ of WT counts. Platelet transfusion reverted macrophage counts back to $92.1 \%$ of WT levels, thus confirming that transfused platelets successfully fostered macrophages extravasation to the site of inflammation (Figure 6B-D). Imaging of fluorescently labeled lavage cells confirmed expression of CD45 and
F4/80, thus verifying their identity as macrophages (Online Supplementary Figure S3A). Moreover, the activation state and reactivity of platelets transfused into $\mathrm{iDTR}^{\mathrm{Plt}}$ was not significantly changed after thioglycollate treatment as compared to those of WT mice (Online Supplementary Figure S3B).

Lastly, we monitored thrombus formation in plateletdepleted $\mathrm{iDTR}^{\mathrm{Plt}}$ mice that received donor platelets from Nbeal2 knockout mice (Nbeal2 $2^{--}$) in a $\mathrm{FeCl}_{3}$-induced vessel injury model. Knockout of Nbeal2 leads to $\alpha$-granule deficiency and thereby severe deficiencies in platelet function and in vivo thrombus formation. ${ }^{13} \mathrm{FeCl} 3$ induced thrombus formation in mesenteric arterioles was monitored intravitally in DT treated iDTR $^{\text {Plt }}$ mice that received either $\mathrm{Nbeal2}^{+/+}$or Nbeal2 ${ }^{-1}$ platelets (Figure 6E). While Nbeal2 platelets were able to form an occlusive thrombus reaching from vessel wall to vessel wall within $21.4 \pm 8.7 \mathrm{~min}$ utes, thrombus formation was severely impaired in mice receiving $\mathrm{Nbeal}^{-\%}$ platelets (Figure $6 \mathrm{~F}-\mathrm{G}$ and Online Supplementary Video S1-2), thus confirming the genetically modified platelet's phenotype ${ }^{14}$ in an otherwise unaltered environment.

\section{Discussion}

In this study, we compared advantages and disadvantages of genetic and antibody-based platelet depletion methods. Antibody-based methods allow controlled adjustment of thrombocytopenia, while the genetic model selectively targets only endogenous megakaryocytes and platelets and is therefore superior when combined with platelet transfusion.

Antibody-based methods directly and indiscriminately target murine platelet epitopes and cause rapid clearance of platelets from the circulation. Contrarily, genetic depletion with the iDTR system works via ablation of megakaryocytes, as a cell type-specific Cre, the PF4iCre4, controls the expression of iDTR. ${ }^{15}$ Thus, megakaryocytes are selectively killed upon DT administration, which prevents endogenous platelet production. Additionally, the decrease of platelet counts corresponded well to the reported lifespan of murine platelets of about 4.8 days ${ }^{10}$ which is in line with the assumption that platelets are naturally used $u^{7,16,17}$ rather than being actively killed by DT.

In general, the expression of a protein that is normally absent in a cell might lead to an alteration of cellular functions. Thus, we found it important to investigate whether iDTR expressing platelets exhibit normal physiological functions, an aspect that is also important for platelet transfusion experiments. To the best of our knowledge, our study is the first to thoroughly analyze platelet function of mice expressing iDTR in megakaryocytes and thus also on the platelet surface. We tested the classical platelet activators thrombin and collagen, as well as agonists that selectively trigger $\mathrm{P}_{2} \mathrm{Y}_{1 / 12}$, PAR4 and GPVI receptors. The fact that iDTR-expressing platelets did not show any noteworthy difference in activation, granule secretion, and aggregation compared to controls supports the notion that these platelets can be considered normal and thus do not cause any unwanted side-effects. As a non-classical function, we analyzed platelet-leukocyteaggregate formation, but were unable to detect any alterations to WT mice. 
A

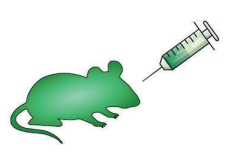

DT

B

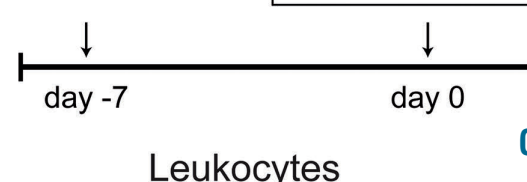

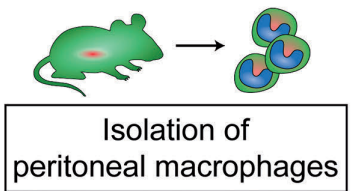

day 3

C

Macrophages
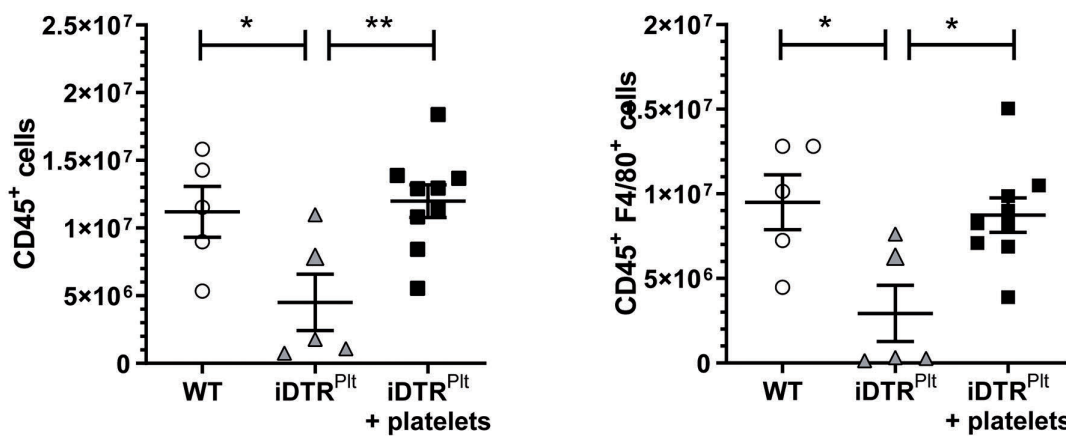

D

WT

iDTR PIt $^{\text {Pl }}$

$\mathrm{iDTR}^{\mathrm{PIt}}+$ platelets
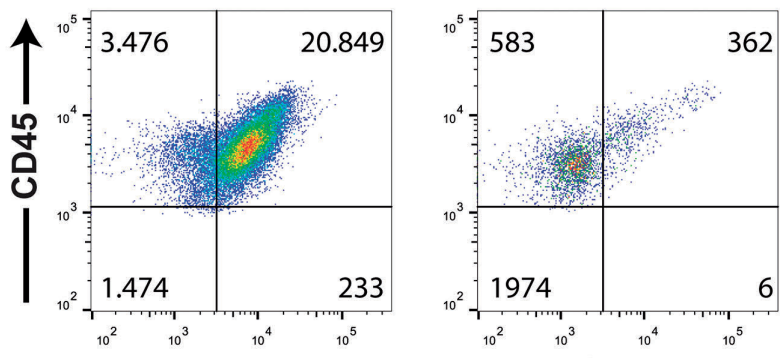

$F 4 / 80$

E

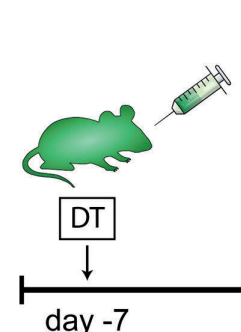

$\mathbf{F}$

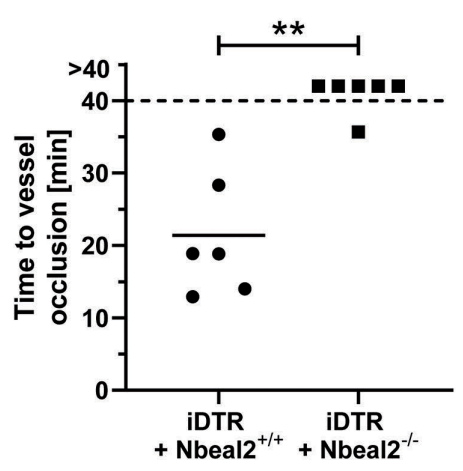

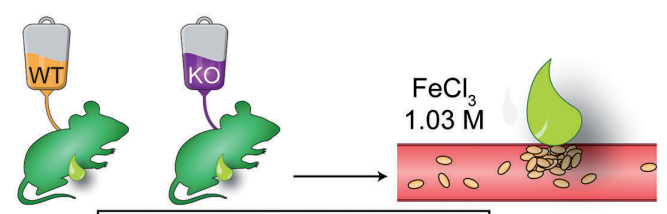

Transfusion and $\mathrm{FeCl}_{3}$ injury

day 0
iDTRPIt

G

+ Nbeal $2^{+/+}$

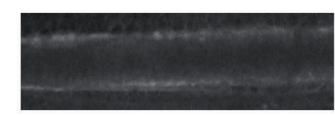

10

$\min$

20

min

40
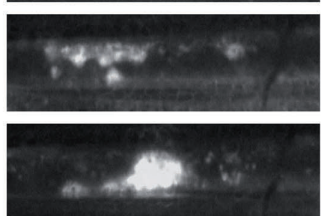

$\min$

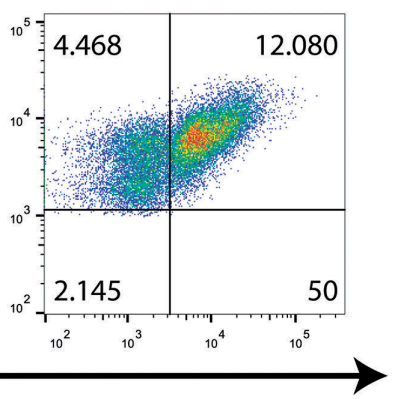

Figure 6. Platelet function and phenotype remains intact after transfusion. (A) Time plan of macrophage recruitment after sterile peritonitis. Seven days after diphtheria toxin (DT) treatment of wild-type (WT) and iDTR $^{\text {Plt }}$ mice, one group of iDTR ${ }^{\text {plt }}$ mice received platelet transfusion, followed by thioglycollate injection. After three days peritoneal macrophages were isolated and quantified. (B) Recruited $\mathrm{CD}_{45^{+}}$cells and (C) $\mathrm{CD}^{2} 5^{+} \mathrm{F} 4 / 80^{+}$cells per mouse $(n=5-9$, mean \pm standard error of the mean [SEM]). (D) Representative blots of peritoneal lavage cells of WT mice, iDTR $^{\text {Plt }}$ mice and iDTR ${ }^{\text {Plt }}$ mice with platelet transfusion. (E) Graphical overview of $\mathrm{FeCl}_{3}$ induced thrombus formation experiments. Seven days after DT treatment of iDTR ${ }^{\text {Plt }}$ mice, one group received $\mathrm{Nbeal} 2^{+}$ and the other Nbeal2 platelets, followed by induction of a thrombus in mesenteric arterioles by topic application of $\mathrm{FeCl}_{3}$. (F) Time to stable vessel occlusion. Each symbol represents a mesenteric arteriole. (G) Representative images of thrombus formation in arterioles $(n=6)$. 
However, MPV was slightly but significantly increased, adding to the contradictory discussion in the literature. While some studies failed to observe a difference during depletion, ${ }^{7}$ we, like others, ${ }^{16}$ detected an increase of MPV during the thrombocytopenic phase. We hypothesize that the few "remaining" platelets were freshly produced by megakaryocytes that were not yet eliminated. These young platelets are reported to have a higher MPV ${ }^{18}$ and are therefore thought to be more reactive. ${ }^{19}$ Clinically, MPV distinguishes between different etiologies of thrombocytopenia. ${ }^{20}$ Thus, it may be a more sensitive biomarker than platelet count in a variety of disorders, yet it is discussed controversially for different diseases. MPV is higher in patients with acute coronary syndrome (ACS), compared to those with non-cardiac chest pain, ${ }^{21}$ associates with poor prognosis after $\mathrm{ACS}^{21}$ and closely correlates

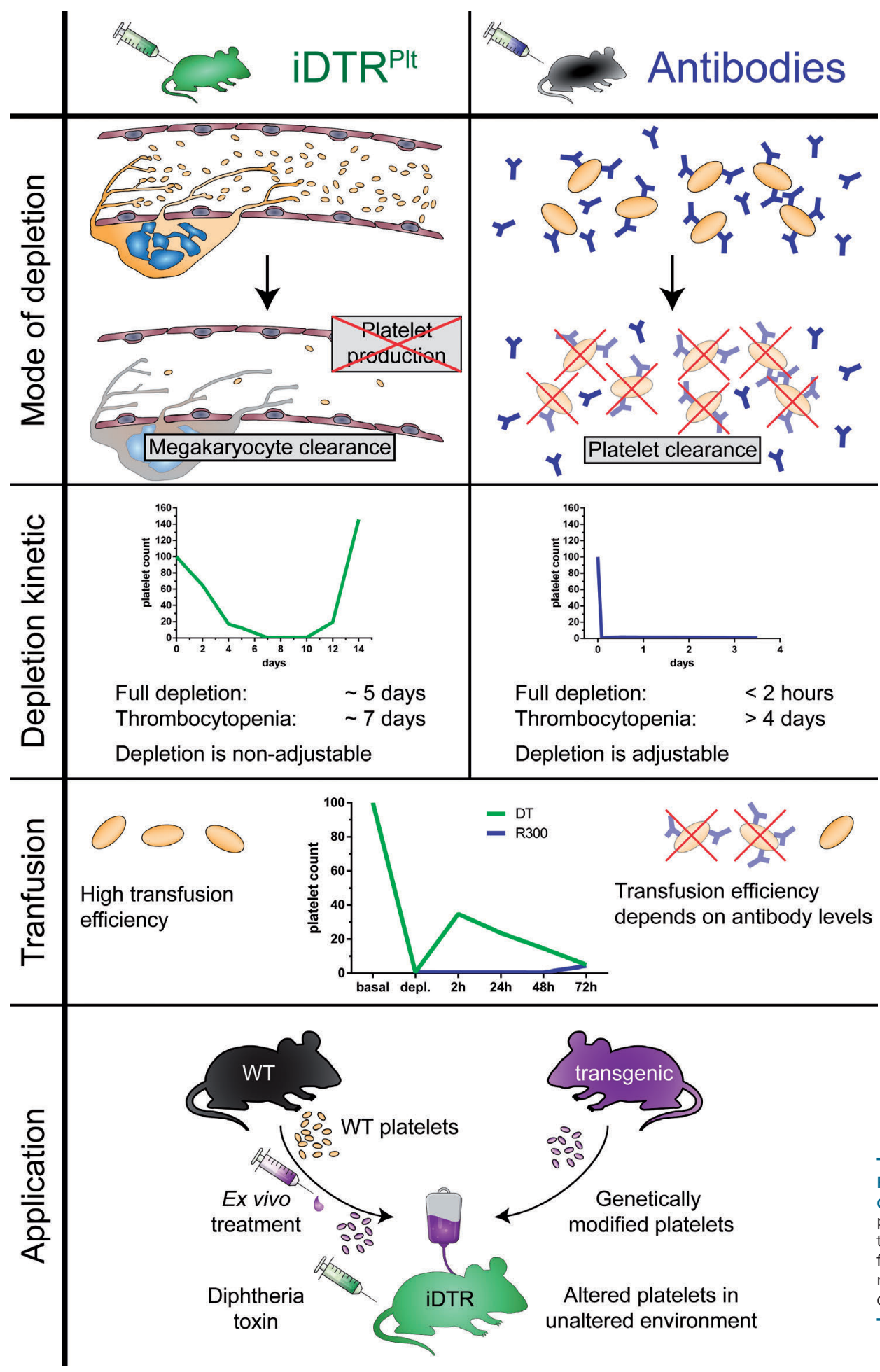

Figure 7. Comparison of platele depletion methods. Depletion principle, depletion kinetic and transfusion efficacy are depicted for the iDTR and antibody-based models, as well as possible applications of the iDTR model. 
with arterial stiffness. ${ }^{22}$ However, diagnosis of coronary artery disease does not associate with MPV. ${ }^{23}$ Further, viruses can alter MPV in both directions, depending on the virus species, ${ }^{24,25}$ and a high MPV can be used to differentiate between gastric ulcer and gastric cancer. ${ }^{26}$

In addition to increased MPV, we also measured increased $\mathrm{P}$-selectin expression of the remaining platelets at day 7 of depletion, an observation also reported by others. ${ }^{7}$ Nevertheless, plasma CXCL4 levels, a surrogate marker for platelet degranulation, were not increased, further underlining that platelets were not activated in circulation.

Like the iDTR system, also antibody-based methods, which induce active and rapid clearance of platelets, did not lead to increased plasma CXCL4 levels or platelet activation. In contrast to the iDTR model, antibody-based methods have the advantage that the amount of injected antibody can be fine-tuned to adjust the degree of thrombocytopenia. The iDTR model completely inhibits platelet production, thus thrombocytopenia cannot be adjusted by titrating DT concentration. This is evident from a rebound effect with rapidly increasing platelet counts after reappearance of megakaryocytes. This phenomenon was also observed by others either after short term treatment, where it was used to study young, reticulated platelets. ${ }^{16}$ In contrast, others were able to keep platelet counts stably low until day 17 using a single injection of 400 ng DT, whereas we observed a rebound at day 14. The reason for this discrepancy is currently unclear. Additionally, continuous depletion of platelets with the iDTR model has been reported for six weeks. ${ }^{27}$ However, at day 12-14 we already observed hematomata and impaired wound healing, thus we decided to anesthetize mice for subcutaneous injections to reduce the risk of injuries. Additionally, we observed that female mice tolerated the treatment better than males. This suggests that females are more suitable as recipients during transfusion experiments, whereas untreated males can be readily used as platelet donors.

However, regarding platelet transfusions, the $\mathrm{iDTR}^{\mathrm{Plt}}$ model shows a significantly better performance than antibody-based models. In the latter, newly transfused platelets are rapidly depleted by an excess of circulating antibodies, which decreases transfusion efficacy and shortens thrombocytopenia by removing the depleting antibodies. This became evident, when endogenous platelet counts of R300 antibody-treated mice increased again at day 3 after transfusion although the dosage of antibody used in our transfusion experiment ensured stable platelet depletion over 3.5 days. In contrast to antibody-based platelet depletion, endogenous platelet counts of $\mathrm{iDTR}^{\mathrm{Plt}}$ mice remained unaffected by the introduction of new platelets, further demonstrating the superiority of the iDTR system.

Finally, we could show that transfused platelets were fully functional by quantifying macrophage recruitment after induction of sterile peritonitis. Platelets are immunomodulatory cells and play a pivotal role in recruiting leukocytes to the site of inflammation. ${ }^{28}$

In conclusion we were able to delineate advantages and disadvantages of different platelet depletion methods (as summarized in Figure 6). While the iDTR model targets platelets indirectly via megakaryocytes, antibody-based models deplete platelets directly. Hence, stable thrombocytopenia is reached with antibodies and anti-mouse thrombocyte serum within 2 or 12 hours, respectively, while the iDTR model takes about five days. Antibodybased methods allow adjustment of platelet counts, depending on the amount of used reagent, whereas the iDTR model results in almost complete thrombocytopenia which may be disadvantageous in certain experimental settings. However, the iDTR model is much more efficient in platelet transfusion compared to antibody based methods, in which freshly transfused platelets and endogenous platelets are scavenged to the same degree.

Altogether, the iDTR model refines platelet transfusion and simplifies its experimental setup, thus reducing the number of required animals. The possibility to transfuse ex vivo-treated platelets or platelets from any global knockout or knockin mouse strain replaces the need to generate lineage-specific conditional mouse strains, further reducing required animal numbers. Moreover, as transfusion of human megakaryocytes ${ }^{29}$ and platelets $^{30}$ into mice has been successfully established, the iDTR model could be potentially useful to evaluate platelet concentrate preparations and storage conditions for clinical applications. Therefore, employing the iDTR-model for platelet depletion and transfusion experiments can facilitate multiple angles of platelet research.

\section{References}

1. Assinger A, Kral JB, Yaiw KC, et al. Human cytomegalovirus-platelet interaction triggers toll-like receptor 2-dependent proinflammatory and proangiogenic responses. Arterioscler Thromb Vasc Biol. 2014;34(4): 801-809.

2. Linden MD, Jackson DE. Platelets: pleiotropic roles in atherogenesis and atherothrombosis. Int J Biochem Cell Biol. Vol. 42. Netherlands: 2010 Elsevier Ltd. 2010:17621766.

3. Starlinger P, Haegele S, Offensperger F, et al. The profile of platelet alpha-granule released molecules affects postoperative liver regeneration. Hepatology. 2016;63(5): 1675-1688.

4. Tiedt R, Schomber T, Hao-Shen H, Skoda
RC. Pf4-Cre transgenic mice allow the generation of lineage-restricted gene knockouts for studying megakaryocyte and platelet function in vivo. Blood. 2007;109(4):15031506.

5. Nagy Z, Vogtle T, Geer MJ, et al. The Gp1ba-Cre transgenic mouse: a new model to delineate platelet and leukocyte functions. Blood. 2019;133(4):331-343.

6. Honjo T, Nishizuka Y, Kato I, Hayaishi $\bigcirc$. Adenosine diphosphate ribosylation of aminoacyl transferase II and inhibition of protein synthesis by diphtheria toxin. J Biol Chem. 1971;246(13):4251-4260

7. Wuescher LM, Takashima A, Worth RG. A novel conditional platelet depletion mouse model reveals the importance of platelets in protection against Staphylococcus aureus bacteremia. J Thromb Haemost. 2015;13(2): 303-313.
8. Yamaizumi M, Mekada E, Uchida T, Okada Y. One molecule of diphtheria toxin fragment $A$ introduced into a cell can kill the cell. Cell. 1978;15(1):245-250.

9. Chang T, Neville DM, Jr. Demonstration of diphtheria toxin receptors on surface membranes from both toxin-sensitive and toxinresistant species. J Biol Chem. 1978;253(19): 6866-6871.

10. Manning KL, Novinger S, Sullivan PS, McDonald TP. Successful determination of platelet lifespan in $\mathrm{C} 3 \mathrm{H}$ mice by in vivo biotinylation. Lab Anim Sci. 1996;46(5):545548.

11. Harkness JE, Wagner JE. The Biology and Medicine of Rabbits and Rodents (ed 3rd). Philadelphia: Lea \& Febiger. 1989

12. Mitruka BM. Clinical biochemical and hematological reference values in normal experimental animals and normal humans 
(ed 2nd ed.). New York: Masson Pub. USA. c1981.

13. Kahr WH, Lo RW, Li L, et al. Abnormal megakaryocyte development and platelet function in Nbeal2(-/-) mice. Blood. 2013; 122(19):3349-3358.

14. Deppermann C, Cherpokova D, Nurden P, et al. Gray platelet syndrome and defective thrombo-inflammation in Nbeal2-deficient mice. J Clin Invest. 2013 July 1. [Epub ahead of print]

15. Buch T, Heppner FL, Tertilt C, et al. A Creinducible diphtheria toxin receptor mediates cell lineage ablation after toxin administration. Nat Methods. 2005;2(6):419-426.

16. Angenieux C, Maitre B, Eckly A, Lanza F, Gachet $\mathrm{C}$, de la Salle H. Time-Dependent Decay of mRNA and Ribosomal RNA during Platelet Aging and Its Correlation with Translation Activity. PLoS One. 2016;11(1):e0148064.

17. Miyachi H, Reinhardt JW, Otsuru S, et al. Bone marrow-derived mononuclear cell seeded bioresorbable vascular graft improves acute graft patency by inhibiting thrombus formation via platelet adhesion. Int J Cardiol. 2018;266:61-66.

18. Balduini CL, Noris P, Spedini P, Belletti S, Zambelli A, Da Prada GA. Relationship between size and thiazole orange fluores- cence of platelets in patients undergoing high-dose chemotherapy. Br J Haematol. 1999;106(1):202-207.

19. Guthikonda S, Alviar CL, Vaduganathan M et al. Role of reticulated platelets and platelet size heterogeneity on platelet activity after dual antiplatelet therapy with aspirin and clopidogrel in patients with stable coronary artery disease. J Am Coll Cardiol. 2008; 52(9):743-749.

20. Schmoeller D, Picarelli MM, Paz Munhoz T, Poli de Figueiredo CE, Staub HL. Mean platelet volume and immature platelet fraction in autoimmune Disorders. Front Med (Lausanne). 2017;4:146.

21. Leader A, Pereg D, Lishner M. Are platelet volume indices of clinical use? A multidisciplinary review. Ann Med. 2012;44(8):805816.

22. Panova-Noeva M, Arnold N, Hermanns MI, et al. Mean platelet volume and arterial stiffness - clinical relationship and common genetic variability. Sci Rep. 2017;7:40229.

23. Ball S, Arevalo M, Wongsaengsak S, Dennis JA, Nugent K. Implications of mean platelet volume in health and disease: A large population study on data from National Health and Nutrition Examination Survey. Thromb Res. 2019;175:90-94.

24. Lv L, Li Y, Fan X, Xie Z, Liang H, Shen T.
HCV coinfection aggravated the decrease of platelet counts, but not mean platelet volume in chronic HIV-infected patients. Sci Rep. 2018;8(1):17497.

25. Assinger A. Platelets and infection - an emerging role of platelets in viral infection. Front Immunol. 2014;5:649.

26. Yun ZY, Li N, Zhang X, et al. Mean platelet volume, platelet distribution width and carcinoembryonic antigen to discriminate gastric cancer from gastric ulcer. Oncotarget. 2017;8(37):62600-62605.

27. Bruns I, Lucas D, Pinho S, et al Megakaryocytes regulate hematopoietic stem cell quiescence through CXCL4 secretion. Nat Med. 2014;20(11):1315-1320.

28. Badrnya S, Schrottmaier WC, Kral JB, et al Platelets mediate oxidized low-density lipoprotein-induced monocyte extravasation and foam cell formation. Arterioscler Thromb Vasc Biol. 2014;34(3):571-580.

29. Wang Y, Hayes V, Jarocha D, et al Comparative analysis of human ex vivogenerated platelets vs megakaryocyte-generated platelets in mice: a cautionary tale. Blood. 2015;125(23):3627-3636.

30. Huo Y, Schober A, Forlow SB, et al Circulating activated platelets exacerbate atherosclerosis in mice deficient in apolipoprotein E. Nat Med. 2003;9(1):61-67. 